\title{
Le tecnologie digitali per la progettazione didattica
}

\section{Pier Giuseppe Rossi}

Università degli Studi di Macerata, Dipartimento di Scienze dell'Educazione e della Formazione

doi: 10.7358/ecps-2014-010-ross

pg.rossi@unimc.it

\section{DIGITAL TECHNOLOGIES FOR LEARNING DESIGN}

\section{Abstract}

The paper aims to analyze how educational technologies are changing the process of instructional design and the processes of personalization and inclusion. The contribution starts by examining the state of the art of instructional design today and how teachers perform their design tasks according to the approach based on "teachers' thinking". The artefacts used in the design process and in the digital author software to support the design itself will be then explored. Starting from the existing scenario, future opportunities are assessed according to the needs created by the complexity of the current context and by the potentialities of today's technologies. The hypothesis is the possibility of creating an artefact for design which can merge two moments that occur before class, the design and preparation of materials. The artefact is meant as a bridge crossing three moments of didactic activity, that is, designing, in-class interaction and documentation. The PROPIT project ("Designing for personalization and inclusion with technologies"), which implies the use of such an artefact, will be briefly described.

Keywords: Design, Digital artifatcs, Enactivism, Personalization, Professionalism.

\section{LA PROGETTAZIONE DELL'AGIRE DIDATTICO}

Per comprendere il ruolo degli artefatti tecnologici nella progettazione didattica il primo passo è indagare come i docenti pianificano la loro azione. Wanlin (2013), confrontando varie ricerche, evidenzia che durante la proget- 
tazione i docenti focalizzano essenzialmente la loro attenzione su tre elementi: obiettivi, contenuti, attività. Due sono le possibili logiche per connettere i tre elementi: (a) si ritiene che pur essendo connessi siano tra loro autonomi e pertanto vadano esaminati in successione, (b) si ritiene che siano in stretta relazione fin dalla fase inziale, come trame interne allo stesso spazio di progetto, e pertanto vadano analizzati con un processo ricorsivo.

Nei modelli classici dell'ID (Instructional Design) i tre elementi sono visti come legati da una logica lineare a waterfall, ovvero dalla definizione degli obbiettivi discende prima la scelta dei contenuti e materiali, e, poi, la struttura delle attività. Così è organizzato ADDIE, il modello di progettazione proposto negli anni sessanta per rispondere all'esigenza di realizzare percorsi formativi di qualità per l'esercito statunitense. ADDIE, il cui acronimo sta per Analysis, Design, Development, Implementation, Evaluation, è il capostipite dei modelli prescrittivi successivi. Dopo ADDIE e sulla scia di ADDIE sono stati realizzati fino ai nostri giorni molte variazioni che però mantengono del capostipite gli elementi caratterizzanti. Nel 1997 un'indagine ha individuato alcune centinaia di modelli (Gustafson \& Branch, 1997).

Dagli anni '90 del secolo scorso vari autori hanno criticato tali modelli per la logica burocratica e la struttura step by step (Jonassen, 1990; You, 1993; Gordon \& Zemke, 2000; Zemke, 2002; Cennano \& Kalk, 2005; Bichelmeyer et al., 2006).

Oggi la maggioranza delle analisi evidenzia la necessità di modelli non prescrittivi che siano in grado di curvarsi sui contesti e che superino la linearità (Branch \& Kopcha, 2014), ma non sempre tali critiche e la proposta di non linearità colgono i motivi più profondi per cui, a nostro avviso, la modellizzazione di $A D D I E$ non corrisponde alle pratiche didattiche e non è applicabile in tutte le situazioni educative.

Il primo fattore, già sottolineato da Bichelmeyer e Tripp nel 1990, è che difficilmente nella fase iniziale del progetto si hanno a disposizione tutti i dati necessari. In base a questa considerazione gli stessi autori hanno proposto il modello basato sul rapid prototyping.

Il secondo fattore, a mio avviso più rilevante, è che i vari elementi (obiettivi, contenuti, strategie e metodologie) non si presentano alla mente del progettista isolati e collegabili con logiche induttive o deduttive, ma già interni a processi complessi, a fili della stessa trama, che poi è l'azione didattica. A supporto di tale analisi possono esserci di aiuto le neuroscienze. Quando Rizzolati e Senigallia (2006) descrivono i processi connessi alla presa di una tazzina sottolineano come la mente parta dalla visione globale dell'azione e non l'analizzi come una successione di movimenti. Così l'avvicinare il braccio e l'afferrare, ma anche l'osservare l'oggetto, il percepirlo, insieme alla finalità del gesto, sono fortemente connessi e fanno tutti parte di un'u- 
nica azione. Tali movimenti sono modellizzati come un unico automatismo ovvero prendere-la-tazzina-per-bere-il-caffè. Il processo vede l'azione come un processo olistico e, se già attuata in precedenza, è simulata mentalmente prima di essere attuata.

Da interviste effettuate con insegnanti si coglie come nel pensare l'azione didattica la loro attenzione si focalizzi sulle attività viste da subito come un intreccio di cose da fare, di contenuti, di obiettivi, di reazioni e di comportamenti degli studenti. L'attenzione agli obiettivi è presente, ma raramente, specialmente nei docenti esperti, da essa prende avvio la progettazione. Laurillard evidenzia come la progettazione possa essere descritta come una narrazione in cui le attività (parla di Learning Activities - LA) costituiscono i nodi principali. Ugualmente Britain (2007 e 2004) parla di LA come ossatura della progettazione. L'azione come nucleo olistico fondante nell'agire didattico e, quindi, nella progettazione è stato approfondito anche in altre sedi (Rossi, 2009; Rivoltella \& Rossi, 2012).

Alcuni autori introducono il concetto di design space (Beadouin-Lafon \& Mackay, 2003) per descrivere la modalità complessa con cui si procede nei processi di progettazione. Secondo tali autori i progettisti creano un design space per ogni specifico progetto: essi esplorano questo spazio, lo espandono o lo riducono e impongono dei vincoli mentre aggiungono ed eliminano degli elementi. All'interno dello spazio si collocano obiettivi, contenuti e strategie. Questo processo è iterativo e si qualifica più per la logica ricorsiva che riduzionista. Il progettista, infatti, raramente inizia da uno specifico elemento e aggiunge poi in modo analitico dettagli sempre più precisi fino alla soluzione finale. Al contrario fin dalla fase iniziale costruisce uno spazio complesso, per poi esplorarlo viverlo e simularlo mentalmente (Gero \& Kannengiesser, 2002). Il docente non analizza analiticamente i vari elementi, ma pensa all'azione didattica come a una scena di un film, in cui i componenti sono tra loro intrecciati. Sceglie tra tutte le azioni pensate quelle che ritiene più efficaci e più sostenibili.

L'aspetto spaziale del fare progettuale ovvero la tendenza a visualizzare, elaborare e modificare mentalmente emerge anche dalle ricerche che partono dal paradigma del pensiero degli insegnanti. Yinger e Clark (1982) hanno mostrato che la visualizzazione dell'attività come si svolgerà nel contesto specifico della propria classe sembra essere un tratto essenziale del processo di progettazione. Altri autori sottolineano come l'artefatto progettuale permetta di memorizzare «l'immagine della lezione» ovvero il flusso delle sequenze (Morine-Dershimer \& Vallance, 1976; Morine-Dershimer, 1979; Smith \& Sendelbach, 1979; Tochon, 1993). 


\section{LE FINALITÀ E I LIVELLI DELLA PROGETTAZIONE}

Alcune indagini sulle finalità della progettazione hanno rilevato che i docenti progettano per organizzare l'attività e semplificare l'azione (Sardo Brown, 1990), per accrescere l'efficacia di un'azione che si svolge in tempi limitati (Bromme, 1982; Creemers \& Westerhof, 1982; Wanlin \& Bodeux, 2006) e per diminuire il livello di incertezza (Clark \& Yinger, 1979; Clark \& Elmore, 1981).

La progettazione diminuisce il livello di incertezza anche grazie alla routinizzazione delle procedure. Il docente, mentre esplicita la propria progettazione, prende consapevolezza, prima, ed elabora, poi, delle routine ovvero procedure utili a controllare e coordinare sequenze di azioni e ad affrontare situazioni che si ripetono frequentemente nella vita della classe. Sicuramente le routine emergono in azione, ma difficilmente durante l'azione il docente riesce a attivare quei processi riflessivi necessari per acquisirne consapevolezza. Sono routine, ad esempio, le modalità con cui si inizia e si conclude la lezione, con cui si correggono i compiti, si affrontano i momenti di stanchezza in classe, o le situazioni di trasgressione delle regole, oppure ancora, si risponde alle domande e agli eventi inattesi o imprevisti. Se l'ID costruisce i propri percorsi sulla stabilità dei contenuti e delle discipline, la progettazione dei docenti nella scuola, si fonda maggiormente sulla risposta ad alcune situazioni che si ripetono nella vita della classe e per le quali ogni docente, in base alla sua filosofia educativa, ha costruito, più o meno consapevolmente, delle routine. Le routine semplificano il processo di progettazione e di organizzazione dell'azione (Yinger, 1980).

Dalle ricerche che analizzano la progettazione partendo dal paradigma del pensiero degli insegnanti (Shulman, 1987) emerge che nel fare progettuale sono presenti differenti livelli: annuale, dei moduli o unità, settimanale, giornaliero (Yinger \& Clark, 1982; Sardo Brown, 1988; Charlier, 1989; Tochon, 1993; Yildirim, 2003; Wanlin, 2013). Per Tillema la natura delle decisioni prese diviene sempre più concreta passando dal livello annuale a quello giornaliero perché maggiormente influenzato dal contesto classe. Se i vari livelli sono tra loro connessi la progettazione della lezione permette di rendere più mirata anche la progettazione annuale (Tillema, 2003).

In altro contributo (Rossi, 2014) si è descritta la pianificazione come insieme interagente di due livelli, un livello macro che contiene la progettazione annuale e i moduli, uno micro che esplode la struttura della lezione. I due livelli si rimandano continuamente e si differenziano non per la granularità, ma per gli elementi su cui si focalizzano: il piano macro è più attento alla struttura disciplinare e alla rete dei contenuti e delle competenze, il livello micro ai mediatori, alle strategie e alle interazione didattiche. 


\section{UNA PRIMA SINTESI}

I docenti hanno tre principali riferimenti durante la progettazione: contenuti, obiettivi e strategie che, però, si intrecciano nell'attività. L'azione è lo spazio pluridimensionale in cui opera e si incorpora la progettazione. La rappresentazione spaziale, esplicita o mentale, accompagna frequentemente i processi progettuali. La progettazione è finalizzata a semplificare l'azione didattica, in particolare quando prevede la gestione di procedure complesse e si sviluppa su più livelli tra loro collegati, ma ciascuno con caratteristiche differenti. Potremmo pertanto affermare che la progettazione è un artefatto semplesso e non solo in quanto la sua funzione è quella di rendere sostenibile la gestione dell'azione didattica, ma anche perché ha il ruolo di anticipare l'attività in classe, prevedendo come essa si svolgerà e quali difficoltà potranno incontrare gli studenti. Inoltre l'azione progettata, come sintesi di finalità, obiettivi, strategie e dinamiche può essere vista come variabile complessa (Berthoz, 2011).

\section{GLi ARTEFATTI PROGETTUALI}

Ma come si reifica la progettazione? La progettazione può essere una traccia presente solo nella mente del docente, oppure essere reificata in uno o più artefatti, cosa che avviene frequentemente per il piano annuale, spesso anche a causa delle richieste istituzionali. Come sottolineano alcuni autori, una progettazione esplicita incide sui percorsi di professionalizzazione degli insegnanti in quanto facilita la riflessione sui processi e una condivisione dei materiali tra i docenti (Britain, 2007). Anche Laurillard individua nella condivisione degli artefatti progettuali tra docenti un passaggio utile allo sviluppo professionale e alla sostenibilità del processo di insegnamento.

Ma che forma hanno gli artefatti progettuali? E, poi, come le tecnologie li stanno modificando?

Occorre precisare il termine artefatto.

Un artefatto è un'entità ideata, progettata e costruita intenzionalmente per raggiungere uno o più scopi (Manzini, 1990; Rizzo, 2000). La caratteristica peculiare degli artefatti è data dall'intenzionalità: essi sono il risultato di azioni intenzionali, piuttosto che involontarie od opportunistiche. (Rossi \& Toppano, 2009, p. 21)

Il termine entità indica sia oggetti materiali, sia concetti. Bereiter (2002) amplia la nozione di artefatto e parla di artefatti concettuali. Il progetto di- 
dattico, se esplicitato, in quanto reificazione di un'idea e supporto all'azione, è un artefatto.

La ricerca in rete ha permesso di recuperare e poi analizzare le progettazioni/programmazioni annuali di alcune decine di scuole italiane o di associazioni di insegnanti. Sono state raccolte progettazione dei tre livelli di scuola e provenienti dalle macro regioni in cui è divisibile l'Italia (Nord-Est, Nord-Ovest, Centro, Sud, Isole). Sono state analizzate 10 progettazioni per ogni categoria che hanno fornito risposte omogenee e tra loro coerenti. L'esame si è concentrato sulla struttura dell'artefatto: nella quasi totalità dei casi l'artefatto utilizzato è una tabella, i cui campi rispondono a logiche simili. Le etichette dei campi non sono sempre le stesse anche se appartengono a categorie simili. Riportiamo alcuni esempi: competenze, conoscenze, abilità; competenza, obiettivi di apprendimento, obiettivi specifici, metodologia; nuclei tematici, conoscenze, abilità; traguardi, obbiettivi, contenuti, indicatori di valutazione. Non molto diverse, sono le programmazioni, anche esse presenti in rete, proposte da riviste specializzate e da comunità di insegnanti. Ad esempio il sito Saperescuola presenta una tabella a due colonne: traguardi per lo sviluppo delle competenze e obiettivi di apprendimento, mentre nel sito Altervista la tabella è composta da nuclei tematici, conoscenze, abilitàcompetenze.

L'uso della tabella per reificare la progettazione ha un limite in quanto non esplicita il legame che connette i vari campi. In ogni riga si hanno varie competenze, varie conoscenze e varie abilità (o altro elemento), ma non si evince come la singola competenza si leghi alla singola conoscenza e alla singola attività. Ancora meno comprensibile è il nesso che lega le strategie/ modalità operative e le competenze/obiettivi/abilità. Molto spesso si scrive che per quel dato modulo si attueranno attività frontali e attività di gruppo, ma senza ancorarle a nessuno specifico processo e senza connettere obiettivo e task. Gli artefatti progettuali tabellari, pertanto non descrivono l'azione, così come nessun legame tra macro e micro progettazione viene esplicitato.

Per comprendere come operano gli insegnanti nella progettazione, seguendo l'approccio del pensiero degli insegnanti, sono stati intervistati 134 docenti di varie scuole e di ordini diversi. L'intervista era condotta da un ricercatore universitario. Alcune domande richiedevano di descrivere le modalità con cui si realizzava la progettazione annuale, altre su come ci si stava preparando per la lezione del giorno dopo. Con la prima domanda si chiede ai docenti se esplicitano le progettazioni. La quasi totalità costruisce la progettazione annuale in forma tabellare, mentre pochissimi costruiscono artefatti per esplicitare la progettazione giornaliera (13\%) e quelli che lo fanno, non la realizzano con continuità $(9 \%)$. Si richiedeva, poi, come e quanto l'attività di progettazione annuale indirizzasse e supportasse le attività 
didattiche quotidiane. I docenti hanno sottolineato la separazione tra lavoro di progettazione annuale e lavoro di aula (68\%), la scarsa utilità del lavoro di progettazione annuale $(76 \%)$ e comunque un utilizzo che non ne giustifica l'impegno e la fatica necessaria per la sua realizzazione (87\%). Alcuni hanno evidenziato come la progettazione annuale abbia fornito un guida generica $(22 \%)$, mentre la maggioranza ha vissuto il lavoro di progettazione come un impegno burocratico e amministrativo che poco ha a che fare con la didattica in classe $(72 \%)$. Gli aspetti positivi della progettazione sono legati ai processi riflessivi che attiva.

Gli stessi insegnanti nel descrivere le modalità con cui pianificano la lezione del giorno dopo hanno parlato di un'attività soprattutto mentale, anche se nella maggioranza dei casi è accompagnata dalla ricerca o produzione di materiali, ovvero documenti che completano l'esposizione dei contenuti proposta dal libro di testo, immagini o video, schede di lavoro. L'uso delle fotocopie per tali materiali è ancora dominante, anche se negli ultimi anni, anche grazie alle LIM, sono sempre più diffusi supporti digitali che oltretutto permettono di ampliare le tipologie di media utilizzabili. A proposito dei materiali digitali molti docenti hanno però sottolineato una difficoltà che spesso incontrano dovuta alla ricerca, selezione, organizzazione e catalogazione sistematica degli stessi. I materiali digitali sono recuperati o da prodotti annessi ai libri di testo o da Internet. Una prima operazione è la selezione non sempre facile dovuta a una qualità non omogenea dei materiali presenti in rete e alla necessità di curvarli alle esigenze del proprio percorso. Inoltre la maggioranza dei docenti non adotta procedure per l'archiviazione e la catalogazione dei materiali stessi.

\section{TeCNOlOgie digitali PER la COSTRUZione DI ARTEFATTI PROGETTUALI}

L'e-learning, il frequente utilizzo di mediatori multimediali e classi sempre più complesse per la presenza di differenti culture, abilità e conoscenze stanno determinando la necessità di una progettazione articolata ed esplicita. Dagli anni '90 del secolo scorso sono stati attivati vari progetti per realizzare standard per la progettazione, per garantirne l'integrazione dei Learning Object negli LMS (Learning Management System ovvero ambienti di apprendimento online) e la condivisione fra differenti docenti. Sono stati anche proposti programmi autore per costruire gli artefatti progettuali così come sono stati proposte modalità per descrivere segmenti di attività che si ripetono con frequenza nell'azione didattica. Tali segmenti sono definiti nella 
letteratura anglofona pattern. Come precisa Laurillard (2012) proprio perché i pattern didattici sono un buon modo per affrontare situazioni complesse e per far circolare le idee,

la comunità che ruota intorno alla progettazione didattica avrà anche bisogno di strumenti che sostengono un'infrastruttura intorno a un pattern. Ci si riferisce a un insieme di strumenti per creare progettazioni e archivi per immagazzinare, organizzare e fornire meccanismi di accesso alle risorse create e di ricerca delle stesse. (ivi, p. 290)

La ricerca nel settore degli ITS ha sicuramente favorito tale elaborazione, integrando nei programmi autore agenti intelligenti con funzione di supporto e di valutazione.

\section{GLI STANDARD}

Uno dei primi standard per la progettazione è stato EML (Educational Modelling Language) realizzato dall'Open University of the Netherlands da cui è derivata nel 2003 la prima versione di LD (Learning Design), prodotto dal consorzio IMS ${ }^{1}$ e connesso agli standard che il consorzio stesso aveva elaborato per LO (Learning Object). Nelle intensioni dei progettisti IMS-LD sarebbe dovuto essere un prodotto pedagogically-free ovvero avrebbe dovuto supportare la progettazione indipendentemente dalla filosofia educativa dell'insegnante. Dopo la prima implementazione sono state realizzate varie release. Nei primi anni il progetto ha creato intorno a sé molto interesse e vari LMS, tra cui Moodle, hanno trasformato la loro struttura per garantire l'interoperabilità. Già alla fine della prima decade del presente millennio gli sviluppi e soprattutto le sperimentazioni sono diminuite di molto. Abbiamo intervistato i principali progettisti di LD e hanno evidenziato due i problemi. Da un lato l'esplosione del web 2.0 ha proposto modalità di lavoro e pratiche comunitarie e partecipative che ben poco avevano a che fare con il modello LD e con la sua complessità. Dall'altro, e sicuramente questo è stato il problema principale, la realizzazione di una progettazione richiedeva molto tempo e presentava molte difficoltà anche operative. In realtà le esigenze tecnologiche, derivanti dall'interoperabilità, hanno prevalso sulle esigenze didattiche e il prodotto finale era laborioso e poco rispondente alle esigenze dei docenti.

Come per i modelli dell'ID, il tempo necessario per realizzare una progettazione con IMS-LD potrebbe essere giustificato solo per corsi attivati

\footnotetext{
1 http://www.imsglobal.org/index.html.
} 
senza modifiche per vari anni, frequentati da un gruppo numeroso di studenti e messi in atto senza una significativa curvatura sul contesto.

McAndrew e Goodyear (2007) elencano i principali problemi insiti nella progettazione supportata da programmi autore digitali in base ai modelli dell'ID: un linguaggio troppo poco ricco per esprimere la complessità dell'agire didattico, un costo alto in termini di tempo e di conoscenze dello specifico linguaggio, un linguaggio informatico complesso che richiede competenze tecnologiche non di base (ivi, p. 93).

\section{Programmi AUtore}

In relazione ai programmi autori Britain, in una review del 2007, elenca alcuni programmi autore per la progettazione: Reload LD editor (costruito in simbiosi con lo standard IMS-LD), MOT , DialogPlus ed $e X e$. Se i primi due editor sono maggiormente adatti a progettisti della formazione e richiedono competenze tecniche avanzate, gli ultimi due non richiedono competenze tecniche avanzate.

Laurillard (2012) in una review suddivide in diverse tipologie gli strumenti per la progettazione. In primis presenta i toolkit per la progettazione didattica e tra questi inserisce LAMS, il toolkit proposto da Conole e Fill (2005), iCOPER (Derntl et al., 2009) e CloudWorks ${ }^{2}$. Elenca poi strumenti dedicati: strumenti interattivi di progettazione (Boyle, 2008; San Diego et al., 2008), una piattaforma personalizzabile per l'apprendimento basato sull' inquiry (Schwartz et al., 1999; Anastopoulou et al., 2009). Infine sottolinea l'esistenza di raccolte di risorse e archivi di LO (Boyle, 2008), raccolte di pattern (Agostinho 2006; Mor \& Winters, 2007; Derntl et al., 2009), resoconti sulle pratiche (Donald et al., 2009), e un wiki per condividere le idee sulla progettazione didattica.

Le tecnologie digitali possono ricoprire efficacemente diversi ruoli di supporto e, data la complessità del processo relativo alla progettazione didattica, tutti questi metodi saranno probabilmente elementi per una infrastruttura di supporto per i docenti. (Laurillard, 2012, p. 293)

Il gruppo di lavoro di cui la ricercatrice è responsabile presso il LKL (London Knowledge Laboratory) ha realizzato il toolkit per la progettazione Learning Designer ${ }^{3}$ (Laurillard \& Masterman, 2009; Charlton \& Magoulas, 2010). Lo strumento permette di progettare due livelli, modulo e lezione, tra loro connessi. La lezione si struttura come successione di attività e il toolkit propone

2 http://cloudworks.ac.uk/cloudscape/view/1882.

3 https://sites.google.com/a/lkl.ac.uk/ldse/. 
una lista di tipologie di LTA (Learning Teaching Activities) da cui attingere per "comporre la lezione». In tal modo permette non solo di costruire una progettazione esplicita, ma supporta il processo. Inoltre per valutare l'artefatto prodotto visualizza con un grafico a torta la presenza nel progetto delle diverse tipologie di apprendimento per comprendere se vi sia stato un uso equilibrato di tali tipologie.

L'uso del tool non richiede nessuna competenza tecnica. Il tool è stato utilizzato, negli anni accademici 2013/14 e 2012/13, da oltre 200 studenti della Laura Magistrale in Scienze Pedagogiche dell'Università degli Sudi di Macerata. Benché il tool sia ancora in una versione beta e vada utilizzato con una serie di accortezze, ha supportato gli studenti nella progettazione soprattutto su due piani: nella implementazione e nella valutazione.

Ultimamente sono apparsi tool basati sulla logica del web 2.0 per la costruzione di percorsi/materiali didattici come ad esempio Blend Space. Sono programmi interessanti in quanto permettono di creare aggregazioni efficaci di oggetti multimediali, quali video, audio, testi e immagini, in modo semplice e rapido. Il limite è la scarsa attenzione alle problematiche dell'insegnamento e della didattica, ovvero supportano in modo limitato il processo di progettazione degli insegnanti. L'attenzione principale è tecnologica e non sempre risulta facile adattare tali artefatti alle esigenze dell'educazione formale.

\section{COME LE TECNOLOGIE POSSONO MODIFICARE LA PROGETTAZIONE}

Lintroduzione delle nuove tecnologie nella progettazione, così come Learning Designer ha evidenziato, permette di supportare l'attività del docente e la sua professionalità, facilitando la realizzazioni di reti di docenti. Tali tool da un lato connettono macro e micro progettazione, dall'altro visualizzano strutture di attività per anticipare la lezione come rete di azioni. Ma, ed è questa la pratica che il contributo vuole introdurre, l'artefatto progettuale potrebbe anche essere utilizzato come mediatore didattico durante la lezione così da supportare l'attività del docente in azione e da migliorare la motivazione, l'orientamento e la partecipazione degli studenti.

\subsection{Le tecnologie come aggregatori}

Nel descrivere gli aspetti innovativi del digitale, in particolare in ambito educativo, nella maggioranza dei casi l'attenzione si focalizza sulla: (1) presenza 
di media diversi, anche tra loro integrati, (2) potenzialità di strumenti per specifiche attività o procedure, quali simulazione, calcolo, costruzione di grafici, automatizzazione di procedure.

Si pone minore attenzione, invece, sul fatto che la presenza delle tecnologie in didattica incide sul ritmo globale e sull'architettura (e visualizzazione) del processo nel suo insieme. Una categoria di artefatti tecnologici è quella degli aggregatori. Se confrontati con oggetti simili non digitali, gli strumenti digitali garantiscono facilità e flessibilità nella predisposizione $\mathrm{e}$ organizzazione, e fluidificano il passaggio tra livelli e tra differenti materiali. Nella progettazione il digitale permette di rendere fluido il passaggio tra macro e micro progettazione e tra la struttura e i materiali. Inoltre l'aggregatore permette di visualizzare spazialmente la progettazione stessa grazie all'utilizzo di diagrammi di flusso o di mappe.

Se il diagramma di flusso o la mappa rendono evidente la logica reticolare e connessionista, il digitale permette l'inserimento nei nodi non solo del concetto e di alcune note relative a tempi e spazi, ma anche dei link ai materiali e ai contenuti che ne sostanziano l'articolazione o l'attività.

Si collega in tal modo il percorso alle attività, la logica della struttura alle attività da svolgere e ai materiali da utilizzare. È questo il valore aggiunto delle tecnologie.

Già oggi sono utilizzati in campo educativo tecnologie che aggregano ed esplicitano la struttura mentre permettono l'accesso ai materiali. Si pensi all'e-portfolio che non solo "mette in ordine» vari contributi e costruisce un elenco ragionato degli stessi, come farebbe un curriculum cartaceo, ma permette di accedere ai documenti e passare dallo schema logico ai materiali. Questo ruolo «ponte» delle tecnologie rende più veloce il passaggio, ma riduce la distanza anche concettuale tra la struttura che assegna un senso e il materiale che dovrebbe supportare tale senso o dovrebbe validarlo. La riduzione della distanza impatta non solo quantitativamente, ma anche qualitativamente sui processi logici sottesi.

Limitato è stato, finora, l'utilizzo degli aggregatori nel campo della progettazione didattica.

La proposta che segue riprende posizioni, precedentemente esplicitate, in particolare quelle di Laurillard e di Britain, e focalizza l'attenzione sulla potenzialità delle tecnologie per realizzare architetture, strutture pertinentirelevance structure (Marton \& Tsui, 2004; Marton \& Pang, 2006) -, pattern e frame work progettuali (Laurillard, 2012) che a un tempo forniscano una rappresentazione del processo e supportino le attività. Quando in didattica si parla di strutture pertinenti, ci si riferisce prevalentemente ai contenuti trattati, ai concetti approfonditi. Non sempre si pensa che la struttura stessa del progetto, ovvero del percorso da attuare, debba essere una struttura rile- 
vante e pertinente e che lo studente debba visualizzarla per percepirla come tale.

L'esplicitazione di tali strutture impatta sia su come il docente prende consapevolezza, anticipa e poi gestisce l'azione didattica, sia su come lo studente partecipa all'azione stessa. Se compito del docente è quello di motivare lo studente e permettergli di generare le proprie articolazioni concettuali e azioni (costruzione e esplicitazione), puntare l'attenzione sulla struttura del corso e della lezione è un elemento alla base dell'allineamento tra gli obiettivi del docente e quelli dello studente.

Un ulteriore vantaggio, che l'uso delle tecnologie potrebbe garantire, è connesso con un altro elemento precedentemente approfondito. Si è detto che molti docenti si raffigurano mentalmente la progettazione con un'immagine spaziale. Molti software permettono la visualizzazione dei progetti con strumenti grafici e danno corpo alle idee, fornendo sia una visione d'insieme sia la possibilità di modificare la granularità. In altro settore Google Map permette di passare dalla visione del pianeta ai particolari di una via e ogni livello mostra dettagli diversi.

\subsection{Le tecnologie come artefatti ponte nei processi}

Altro effetto dell'introduzione delle tecnologie nei processi produttivi è stato quello di costruire artefatti ponte tra le varie fasi dalla progettazione alla realizzazione del prodotto. Due esempi per esplicitare il concetto. Oggi i giornalisti producono i loro articoli già in un formato adatto alla pubblicazione senza passare attraverso la manipolazione del linotipista, così come il prodotto di chi impagina va direttamente in stampa. Ugualmente lo schizzo dell'architetto o del designer, con la digitalizzazione, diviene un artefatto utilizzabile, grazie a stampanti 3D, per costruire i modelli e, poi, per elaborare le schede di lavorazione e i disegni esecutivi (Ciccarelli, 2002; Rossi \& Toppano, 2009). Figure come quella del modellista sono divenute obsolete e in vari settori si passa in modo diretto dal disegno al modello e dal modello al prototipo. Tali cambiamenti hanno richiesto che il singolo professionista acquisisse competenze differenti e divenisse una figura ibrida. Altre figure ibride introdotte dalle tecnologie digitali e in particolare dal web 2.0 sono i prosumer che superano, in parte, la divisione tra chi costruisce e chi utilizza prodotti digitali.

Se volgiamo lo sguardo al settore della progettazione didattica si percepisce immediatamente come il vento del web 2.0 non sia ancora percepibile e che l'artefatto progettuale non abbia acquisito ancora quella longitudinalità dalla fase iniziale preparatoria alla fase dell'azione didattica. Rimane uno 
strumento in mano al docente e non viene usato esplicitamente in classe durante la lezione con gli studenti e, poi, nella documentazione. In ambito educativo sono stati scarsamente presenti, come hanno evidenziato Carroll, Chin, Rosson e Neale (2000) modalità operative quali l'User Centered Approach e il Partecipatory Design (Willis \& Wright, 2000; Krug, 2005; CarrChellman, 2007; Baek et al., 2008).

\section{UN ARTEFATTO PER LA PROGETTAZIONE DIDATTICA}

In base alle precedenti analisi, relative al modo di progettare degli insegnati, alla necessità di una progettazione esplicita e al supporto potenziale delle tecnologie, è stata implementata la seguente ipotesi progettuale: realizzare un artefatto digitale che reifichi e rappresenti la struttura della pianificazione didattica, supporti il docente nella fase di progettazione, sia utilizzabile come mediatore didattico durante la lezione per fornire uno scaffolding all'attività dei docenti e un orientamento agli studenti.

Le ipotesi di ricerca sono pertanto:

1. Le tecnologie forniscono alla progettazione didattica strumenti per costruire strutture pertinenti e flessibili anche grafiche, per meglio delineare lo spazio concettuale dell'agire educativo, impattando sulla consapevolezza progettuale degli insegnanti.

2. La struttura ipertestuale garantisce una connessione tra macro e micro progettazione.

3. La creazione dell'artefatto digitale impatta sull'allineamento tra docenti e studenti e sull'autonomia degli studenti.

4. La struttura ipertestuale e la visualizzazione in classe rendono sostenibile la personalizzazione e l'inclusione.

Sintetizzando quanto fino ad ora affermato, la progettazione ha come nodi centrali le attività, si avvale di raffigurazioni spaziali, si struttura su più livelli, tra loro connessi, ma ciascuno con una propria logica. Un artefatto progettuale esplicito semplifica l'azione e permette al docente di gestirla e allo studente di anticiparla e orientarsi. I principali programmi autore fino a ora utilizzati realizzano artefatti di supporto visibili ai soli insegnanti, mentre molti prodotti del web 2.0 esplicitano il prodotto finale, ma non accompagnano i processi di progettazione. Inoltre la complessità della classe pone il problema della personalizzazione e dell'inclusione (Giaconi \& Rossi, 2014) che, insieme alla disponibilità di molti materiali anche multimediali digitali, richiedono strumenti a supporto della progettazione, in quanto il solo utilizzo della memoria può risultare poco efficace e dispersivo. Infine progettare 
richiede tempo. Sulla strada della sua sostenibilità un passaggio potrebbe consistere nel connettere il lavoro per ideare il percorso alla predisposizione dei materiali didattici.

Per affrontare queste problematiche è stato promosso il progetto PROPIT (Progettare per la personalizzazione e l'inclusione con le tecnologie).

Il progetto prevede di costruire i vari livelli progettuali con diagrammi di flusso tra loro connessi: il diagramma della progettazione annuale i cui nodi sono i moduli e/o le unità di apprendimento, il diagramma dei moduli/ unità i cui nodi sono le lezioni, il diagramma delle lezioni i cui nodi sono le attività. In ogni nodo attività sono presenti alcune note con le informazioni che gli insegnanti reputano importanti (durata, obiettivi, competenze, focalizzazioni) e il link ai materiali da utilizzare, link che rimandano o a Internet (ad esempio nel caso di video presenti in YouTube), oppure a prodotti realizzati dagli insegnanti stessi (schede di approfondimento, compiti, proposte di attività) collocati in un cloud (nelle nostre sperimentazioni utilizziamo Google Drive che permette anche attività collaborative tra studenti).

I diagrammi pertanto reificano delle reti che collegano diacronicamente i livelli diversi e sincronicamente i materiali utilizzati a lezione, divenendo in tal modo uno strumento per l'insegnante nella gestione dell'azione didattica.

I diagrammi sono visualizzati in classe tramite LIM e utilizzati come ossatura della lezione, per favorire la consapevolezza degli studenti sulla struttura del percorso. La connessione tra mappe di livelli diversi permette con un solo click di passare dalla focalizzazione della lezione del giorno alla visione della mappa annuale evidenziando dove sia posizionato nel percorso globale il frammento di conoscenza analizzato nella singola lezione.

La presenza dell'artefatto in classe e la facile manipolazione che caratterizza gli artefatti digitali permette che gli stessi durante la lezione siano implementati aggiungendo nodi e materiali frutto degli eventi che caratterizzano l'azione didattica. In tal modo si garantisce la flessibilità della progettazione e la possibilità di inglobare l'evento.

L'artefatto relativo alla lezione prevede che alcuni nodi siano posti diacronicamente (in successione). Ma in alcuni casi i nodi potrebbero essere posti in parallelo così da essere utilizzati nello stesso intervallo di tempo da gruppi diversi di studenti. I nodi presentano attività alternative tra loro e la differenza potrebbe dipendere dal mediatore utilizzato, dal compito assegnato, dall'approfondimento richiesto. La sincronia impatta con l'inclusione e la personalizzazione creando percorsi plurimi gestibili anche in autonomia dagli studenti.

Le tecnologie digitali hanno un ruolo strategico in PROPIT. La loro struttura reticolare facilita la rappresentazione del processo, il linguaggio digitale permette di aggregare prodotti multimediali, l'utilizzo del diagramma 
tramite LIM garantisce una visione uno a molti, la presenza dei materiali nel cloud supporta la possibilità per il singolo studente di vedere mappe e prodotti sul proprio tablet o $P C$ sia in classe sia a casa, e di operare sullo stesso. Il supporto informatico garantisce la flessibilità e la manipolazione in tempo reale.

\section{LE ATTIVITÀ SPERIMENTALI, I PRIMI RISULTATI SPERIMENTALI E PROBLEMI EMERSI}

Il progetto PROPIT, coordinato dal gruppo $\mathrm{EDIT}^{4}$, è stato attivato dal Dipartimento di Scienze della Formazione, dei Beni Culturali e del Turismo dell'Università degli Studi di Macerata e vede coinvolti docenti dell'ateneo maceratese, dell'Università degli Studi del Molise e dell'Università «Suor Orsola Benincasa» di Napoli. Al progetto hanno aderito scuole di varie regioni e di differenti livelli ${ }^{5}$.

Il progetto adotta la metodologia della ricerca collaborativa tra scuola e università (Desgagnè $\&$ Larouche, 2010). Le competenze progettuali dei docenti della scuola e la loro esperienza didattica e disciplinare si confrontano con le proposte del progetto, modificandole e curvandole alle esigenze dei contesti, e con le metodologie di ricerca per la validazione del percorso, avanzate dai ricercatori universitari.

Nel periodo febbraio-maggio 2014 sono stati realizzati singoli moduli per mettere a punto il prototipo e testarlo nelle classi. Da settembre sono state predisposte progettazioni annuali che verranno attuate nelle classi durante l'anno scolastico 2014/15.

L'esperienza fino ad ora realizzata ha confermato alcune ipotesi e evidenziato alcuni problemi.

Come è emerso da alcuni incontri tra ricercatori universitari e insegnanti delle scuole, la costruzione del diagramma aumenta la consapevolezza dei docenti, permette una gestione più precisa dei tempi e una maggiore tranquillità nella gestione della classe. Inizialmente alcuni docenti avevano paventato che l'uso delle mappe richiedesse tempo. Un breve periodo di rodaggio ha garantito un uso trasparente delle tecnologie. I problemi iniziali hanno riguardato la didattica e la necessità di passare nella progettazione da una logica per obbiettivi a una logica per attività.

4 http://www.editlab.it/.

5 Tra gli altri: IC Torre (PN), LS Rivoli (TO), IC Sassoferrato (AN), IC Fermi (MC), IC Caldarola (MC), IPSIA SBT (AP), IC Pomigliano d'Arco (NA). 
I maggiori problemi derivano dalle tecnologie. Per la costruzione delle mappe è stato adottato VUE (Visual Understanding Environment), prodotto dalla Tufts University di Boston, fruibile gratuitamente. È di facile gestione, personalizzabile e presenta una struttura essenziale. Nei nodi è possibile collocare info e materiali. Ha però due limiti. In primis non presenta quelle funzioni che, ad esempio, possiede Blend Space per poter inserire con un semplice drag and drop materiali dalla rete. Costringe i docenti a copiare indirizzi dalla rete e a incollarli nei nodi. Inoltre, e questo è per ora il limite principale, non vi è l'app per Android e Apple e pertanto non è utilizzabile in quelle classi che hanno adottato il tablet.

Il problema è dunque quello che normalmente incontrano i docenti quando utilizzano le tecnologie: la mancanza di specifici tool per la didattica. Per superare tale problema Laurillard suggerisce che siano i docenti a proporre le caratteristiche e a guidare la progettazione. Proprio in questa direzione è in atto un progetto per la realizzazione di un artefatto tecnologico che possa soddisfare i requisiti evidenziati dalla sperimentazione e dalla ricerca sulla progettazione.

Nel prossimo anno l'indagine si focalizzerà sia su aspetti che riguardano lo studente, sia su aspetti che riguardano il docente. In relazione a questo ultimo si verificherà la sostenibilità con cui riesce a gestire la personalizzazione e l'inclusione, e gli effetti dell'esplicitazione della progettazione sulla professionalità e sulla condivisione. In relazione allo studente, si vuole indagare come la struttura incida sul metodo di studio, sulla partecipazione e, soprattutto, sull'autonomia con cui gestisce il percorso e sulla loro capacità di orientarsi e avere una visione complessa e complessiva del percorso.

\section{Conclusione}

Una delle osservazioni più comuni, tra i docenti che attuano la sperimentazione, è relativa a come il progetto possa favorire la condivisione e la discussione tra colleghi e incidere sulla consapevolezza professionale. Il nodo della professionalità docente e l'importanza del confronto e della condivisione emergono come bisogno dei docenti e come strumenti utili alla qualità del proprio lavoro. Il legame tra professionalità e progettazione didattica è importante, soprattutto oggi. Se Laurillard intitola uno dei suoi saggi più recenti Teaching as a design science non è un caso. La ricercatrice vede come nodi centrali della professionalità docente, la progettazione didattica, la capacità di anticipare e poi regolare la complessità dell'agire didattico. Visioni riduttivistiche, che fanno riferimento a un insegnante efficace o forniscono le ricette su quello che in assoluto funziona, sono oggi quanto mai inutili per 
affrontare realtà complesse che richiedono processi personalizzati e inclusivi, progettati in base a un'ampia conoscenza delle strategie disponibili e alla capacità di costruire percorsi curvati sul contesto.

L'introduzione delle tecnologie nella scuola, finora maggiormente pensata in direzione della multimedialità o di artefatti per specifiche attività, potrebbe supportare il processo ricorsivo progettazione-azione-documentazione. Un artefatto progettuale digitale, che svolga il ruolo di ponte tra le diverse fasi dell'agire didattico, garantisce la ricorsività: è l'artefatto progettuale, è il mediatore che in classe descrive la struttura dell'azione, è lo strumento per la documentazione e la riflessione finale.

Le idee ci sono, le tecnologie potrebbero permetterlo, ma non abbiamo a oggi un artefatto perfettamente performante con le caratteristiche sopra descritte. Lo sviluppo attuale delle tecnologie non sempre è adeguato alle richieste dei contesti formali dell'apprendimento, mentre è molto più attento ad altri settori e ai contesti informali. Come Laurillard sostiene, finora nella scuola sono state importate tecnologie pensate per altri settori: così è per Power Point, per gli strumenti di comunicazione e per la scrittura. Il salto di qualità avverrà se i docenti, gli esperti della formazione, opereranno con gli informatici nella progettazione di nuovi strumenti, per la didattica e pensati per la didattica. Richiede un livello diverso di progettazione interdisciplinare degli artefatti e una consapevolezza nuova della professionalità docente. Questo è lo step che ci attende.

\section{RIFERIMENTI BIBLIOGRAFICI}

Agostinho, S. (2006). The use of a visual learning design representation to document and communicate teaching ideas. Paper presented at the 23rd Annual Ascilite Conference.

Anastopoulou, S., Sharples, M., Ainsworth, S., \& Crook, C. (2009). Personal inquiry: Linking the cultures of home and school with technology mediated science inquiry. In N. Pachler \& J. Seipold (Eds.), Mobile learning cultures across education, work and leisure. Proceedings of the 3rd WLE Mobile Learning Symposium. London: Work-Based Learning for Education Centre.

Baek, E., Cagiltay, K., Boling, E., \& Frick, T. (2008). User-centered design and development. Handbook of Research on Educational Communications and Technology, 14(1), 659-670.

Beadouin-Lafon, M., \& Mackay, W. (2003). Prototyping tools and techniques. In J. Jacko \& A. Sears (Eds.), The human-computer interaction handbook: Fundamentals. Evolving technologies and emerging applications (pp. 1006-1031). Mahwah, NJ: Lawrence Erlbaum Associates Publishers. 
Bereiter, C. (2002). Education and mind in the knowledge age. Hillsdale, NJ: Lawrence Erlbaum Associates Publishers.

Berthoz, A. (2011). Sempolessità. Torino: Codice.

Bichelmeyer, B., Boling, E., \& Gibbons, A. (2006). Instructional design and technology models. Their impact on research, practice and teaching in IDT. In M. Orey, J. McLendon, \& R. Branch, Educational media and technology yearbook. Westport: Libraries Unlimited.

Bichelmeyer, B., \& Tripp, S. D. (1990). Rapid prototyping: An alternative. Educational Technology Research and Development, 38, 31.

Boyle, T. (2008). The design of learning objects for pedagogical impact. In L. Lockyer, S. Bennett, S. Agostinho, \& B. Harper (Eds.), The handbook of research on learning design and learning objects: Issues, applications and technologies. Hershey: Information Science Reference.

Branch, R. M., \& Kopcha, T. J. (2014). Instructional design models. In J. Spector, Handbook of research on educational communications and technology. New York: Springer.

Britain, S. (2004). A review of learning design: Concept, specification and tools. http:// scholar.google.com/url?sa=U\&q=www.jisc.ac.uk/uploaded_documents/ ACF1ABB.doc (consulted 10/08/2006).

Britain, S. (2007). Learning design systems: Current and future developments. In H. Beetham \& R. Sharpe (Eds.), Rethinking pedagogy for a digital age (pp. 103-115). New York: Routledge.

Bromme, R. (1982). How to analyze routines in teachers' thinking processes during lesson planning. Paper presented at Annual Meeting of the American Educational Research Association. http://files.eric.ed.gov/fulltext/ED223546.pdf.

Carr-Chellman, A. A. (2007). User Design. Mahwah, NJ: Lawrence Erlbaum Associates Publishers.

Carroll, J. M. (2000). Making use: Scenario-based design of human-computer interactions. Cambridge, MA: MIT Press.

Carroll, J. M., Chin, G., Rosson, M. B., \& Neale, D. C. (2000). The development of cooperation: Five years of participatory design in the virtual school. In Processes, practices, methods, and techninques (pp. 239-251). Proceedings of the Conference on designing interactive systems. London: ACM.

Cennano, K., \& Kalk, D. (2005). Real world instructional design. Belmont: Thomson Wadsworth.

Charlier, E. (1989). Planifier un cours, c'est prendre des décisions. Bruxelles: De Boeck. Ciccarelli, N. (2002). Progettare nell'era digitale. Venezia: Marsilio.

Clark, C. M., \& Elmore, J. L. (1981). Transforming curriculum in mathematics, science, and writing: A case study of teacher yearly planning. Research Series, 99.

Clark, C. M., \& Yinger, R. J. (1979). Three studies of teacher planning. Research Series, 55. 
Charlton, P., \& Magoulas, G. D. (2010). Autonomic computing and ontologies to enable context-aware learning design. Paper presented at 22nd International Conference on tools with artificial intelligence, Arras.

Conole, G., \& Fill, K. (2005). A learning design toolkit to create pedagogically effective learning activities. Journal of Interactive Media in Education. http://jime. open.ac.uk/2005/08.

Creemers, B. P., \& Westerhof, K. (1982). Routinization of instructive and management behavior of teachers. Haren: Educational Research Institute in the North.

Derntl, M., Neumann, S., \& Oberhuemer, P. (2009). Report on the standardized description of instructional models. ECP 2007 EDU 417007.

Desgagné, S., \& Larouche, H. (2010). Quand la collaboration de recherche sert la légitimation d'un savoir d'expérience. Recherche en Éducation - Hors Série, 1, 7-18.

Donald, C., Blake, A., Girault, I., Datt, A., \& Ramsey, E. (2009). Approaches to learning design: Past the head and the hands to the HEART of the matter. Distance Education, 30(2), 179-199.

Gero, J., \& Kannengiesser, U. (2002). The situated function-behaviour-structure framework. In J. Gero (Ed.), Artificial intelligence in Design '02 (pp. 89-104). Dordrecht: Kluver Academic Publishers.

Giaconi, C., \& Rossi, P. G. (2014). Progetto PROPIT: progettare per la personalizzazione e l'inclusione con il supporto delle tecnologie. SIM, 2, 42-44.

Gordon, J., \& Zemke, R. (2000). The attack on ISD. Training, 37(4), 42-53.

Gustafson, K. L., \& Branch, R. M. (1997). Revisioning models of instructional development. Educational Technology Research and Development, 45(3), 73-89.

Jonassen, D. (1990). Thinking technology: Chaos in instructional design. Educational Technology, 30(2), 32-34.

Krug, S. (2005). Don't make me think: A common sense approach to web usability. Indianapolis: Pearson Education.

Laurillard, D. (2012). Teaching as a design science. London: Routledge.

Laurillard, D., \& Masterman, E. (2009). TPD as online collaborative learning for innovation in teaching. In O. Lindberg \& A. D. Olofsson (Eds.), On line learning communities and teaching professional development: Methods for improved educational delivery. Berlin: Springer, 230-246.

Manzini, E. (1990). Artefatti. Verso una nuova ecologia dell'ambiente artificiale, Milano: Domus Academy.

Marton, F., \& Pang, M. F. (2006). On some necessary conditions of learning. Journal of the Learning Science, 193-220.

Marton, F., \& Tsui, A. B. M. (2004). Classroom discourse and the space of learning. Mahwah, NJ: Lawrence Erlbaum Associates Publishers. 
McAndrew, P., \& Goodyear, P. (2007). Representing practitioner experiences through learning design and patterns. In H. Beetham \& R. Sharpe (Eds.), Rethinking pedagogy for a digital age: Designing and delivering e-learning (pp. 92-102). London: Routledge.

Mor, Y., \& Winters, N. (2007). Design approaches in technology-enhanced learning. Interactive Learning Environments, 15(1), 61-75.

Morine-Dershimer, G. (1979). Teacher plan and classroom reality. East Lansing: Michigan State University.

Morine-Dershimer, G., \& Vallence, E. (1976). Teacher planning. San Francisco: Far West Laboratory.

Rivoltella, P. C., \& Rossi, P. G. (2012). L'agire didattico. Brescia: La Scuola.

Rizzo, A. (2000). La natura degli artefatti e la loro progettazione. Sistemi Intelligenti, 12(3), 437-52.

Rizzolati, C., \& Sinigaglia, G. (2006). So quel che fai. Il cervello che agisce e i neuroni specchio. Milano: Raffaello Cortina.

Rossi, P. G. (2009). Didattica enattiva. Milano: FrancoAngeli.

Rossi, P. G. (2014). Tra macro e micro progettazione: che significa progettare per attività? SIM, 2, 48-50.

Rossi, P. G., \& Toppano, E. (2009). Progettare nella società della consocenza. Roma: Carocci.

San Diego, J. P., Laurillard, D., Boyle, T., Bradley, C., Ljubojevic, D., Neumann, T., $\&$ Pearce, D. (2008). Towards a user-oriented analytical approach to learning design. ALT-J, 16(1), 15-29.

Sardo Brown, D. (1988). Twelve middle school teachers' planning. Elementary School Journal, 89, 69-87.

Sardo Brown, D. (1990). Experienced teachers planning practice: A US survey. Journal of Education for Teaching, 16(1), 57-71.

Shulman, L. (1987). Knowledge and teaching: Foundations of the new reform. Harvard educational Review, 1, 1-21.

Smith, E. L., \& Sendelbach, N. B. (1979). Teacher intention for science instructionand their antecedents in programs materials. San Francisco: AERA.

Schwartz, D., Brophy, S., Lin, X., \& Bransford, J. (1999). Software for managing complex learning: Examples from an educational psychology course. Educational Technology. Research and Development, 47(2), 39-59.

Tillema, H. (2003). Categories in teacher planning. In M. Kompf \& P. M. Denicolo, Teacher thinking twenty years on: Revisiting persisting problems and advances in education (pp. 61-69). Lisse: Swets \& Zeitlinger.

Tochon, F. (1993). L'enseignant(e) expert(e). Paris: Nathan.

Wanlin, P. (2013). La pensée des enseignants lors de la planification de leur enseignement. Revue Française de Pédagogie. http://rfp.revues.org/1294. 
Wanlin, P., \& Bodeux, C. (2006). Les processus de pensée des enseignants durant la planification et l'implémentation de leur enseignement. Actes du Colloque International de l'Association pour le Développement des Méthodologies d'Évaluation en Éducation. Luxembourg.

Willis, J., \& Wright, K. E. (2000). A general set of procedures for constructivist instructional design: the new R2D2 model. Educational Technology, 40(2), 5-20.

Yildirim, A. (2003). Instructional planning in a centralized school system: Lessons of a study among primary school teachers. International Review of Education, $49(5), 525-543$.

Yinger, R. J. (1980). A study of teacher planning. The Elementary School Journal, 80, 107-127.

Yinger, R. J., \& Clark, C. M. (1982). Understanding teachers' judgments about instruction: The task, the method, and the meaning. Research Series, 121.

You, Y. (1993). What can we learn from chaos theory? An alternative approach to instructional systems design. Educational Technology Research and Development, 41(3), 17-32.

Zemke, R. A. (2002). A hard look at ISD. Training Magazine, 39(2), 26-35.

\section{Riassunto}

Il contributo vuole analizzare come le tecnologie dell'educazione stiano modificando il processo di progettazione didattica e i processi di personalizzazione e inclusione. Il contributo seguirà il seguente percorso. Si partirà dallo studio dello stato dell'arte della progettazione didattica e dall'analisi di come oggi progettano gli insegnanti, sulla base di indagini realizzate con l'approccio del pensiero degli insegnanti. Saranno, poi, esplorati gli artefatti utilizzati oggi nella progettazione e i programmi autore digitali per supportare la progettazione stessa. Partendo dall'esistente si cercherà di indagare quali potrebbero essere le prospettive future in funzione delle esigenze poste dalla complessità del contesto attuale e dalle potenzialità delle attuali tecnologie. L'ipotesi è quelle di realizzare un artefatto progettuale che fonda $i$ due momenti che anticipano la lezione (la progettazione e la preparazione di materiali), e che attraversi i tre momenti dell'agire didattico, ovvero la progettazione, l'interazione in classe e la documentazione. Si descriverà sinteticamente il progetto PROPIT ("Progettare per la personalizzazione e l'inclusione con le tecnologie») che prevede il supporto di tale artefatto e i primi risultati sperimentali.

Parole chiave: Artefatti digitali, Design, Didattica enattiva, Personalizzazione, Professionalità. 